\title{
Facial Soft Tissue Thickness in North-West of Iran
}

\author{
Johari Masoume $^{1}$, Esmaili Farzad ${ }^{1}$, Markazi Moghaddam Mohaddeseh ${ }^{2 *}$
}

1. Associated professor of oral \& maxillofacial radiology, dental faculty, Tabriz University of medical science

2. Post graduate student of oral \& maxillofacial radiology, dental faculty, Tabriz University of medical science

\begin{abstract}
Introduction: To obtain correct facial reconstruction of human skull it is necessary to know the average facial soft tissue thickness of specific sites of the face. This requires establishing a database of soft tissue thickness related to ethnicity, weight and height. The present study determined the facial soft tissue thickness in the different landmark points on the CBCT images of patients.

Materials and methods: In this study, the CBCT images of 250 patients were observed and facial soft tissue thickness (FSTT) was measured in 14 anatomical land marks. After scan, height and weight of the patients were collected and BMI was calculated. Patients were divided into three groups of low, normal and high BMI. FSTT was compared with BMI.

Results: In some land marks, significant differences were found between underweight, normal and overweight patients. In the most cases, facial soft tissue thickness increased with increased BMI. Only inferior malar point thickness decreased by increasing BMI.

Conclusion: These data can be used for facial reconstruction purposes in Iranian population; however, slight differences between studied population and other subgroup races must be considered for the accurate reconstruction.
\end{abstract}

Key words: Soft tissue thickness; Facial reconstruction; Cone Beam Computed Tomography

\section{Introduction}

Reconstruction of faces on skulls is commonly used for individual identification in forensic medicine. The knowledge of the average facial soft tissue thickness of specific sites is essential to the correct facial reconstruction of human face. To reach this goal, a thorough database of soft tissue thickness correlating to age, sex, race and ethnicity is prerequisite. Following the first introduction in the late $19^{\text {th }}$ century, some technics have been developed (1) including radiography, ultrasound, $\mathrm{MRI}, \mathrm{CT}$ and Cone Beam Computed Tomography (CBCT).

\section{Corresponding author:}

Markazi Moghaddam, Mohaddeseh

Post graduate student of oral \& maxillofacial radiology, dental faculty, Tabriz University of medical science.

Corresponding author

Email: m.mmoghadam@yahoo.com

Receive date: 2014-08-16| Accept date: 2014-08-29| Publish date: 2014-09-19

DOI: 10.7575/aiac.abcmed.15.03.01.08

\section{Al}


Radiography and CT may be harmful because of radiation exposure. Ultrasound can result in inaccuracy in the measurement due to the rather complicated detection of the transition point between bone and soft tissue (2). Recently developed CBCT, however, enables images to be obtained with the subjects in an upright position. In addition, radiation dose in CBCT is much less than multi-slice CT (3). Previous studies have suggested that there is significant variation in facial tissue thickness between different populations. Databases of facial soft tissue thickness are available for the Turks, Indians, and Slovak (2, 4, 5). Some studies suggest that different body type (overweight, normal, underweight) plays an important role in facial reconstruction. As we know, there is no study on Iranian population using CBCT; while, CBCT is the choice specialized imaging technic in head and neck region and in dentistry. The aim of this study was to collect a database of average FSTT in 14 anatomical landmarks on the bases of body mass index (BMI) in Iranian-Azeri race.

\section{Materials and methods}

In this study, 250 pateints who were scheduled for head CBCT due to various purposes were recruited and studied in the radiology department of Tabriz Faculty of Dentistry, Tabriz, Iran. Exclusion criteria were inflamatory or infected lesions, soft tissue and bone masses, cystic lesions and congenital anomaleis. After scan, demographic characteristics of the pateints such as weight and height were recorded. Body mass index was calculated by placing the height and weight at the formula $\left(\mathrm{kg} / \mathrm{m}^{2}\right)$. axial, coronal and sagitall images were analysed to measure 14 landmarks by an oral and maxillofacial radiologist (Fig. 1). NewTom VG CBCT with a cone beam of 360 degree rotation, $0.127 \mathrm{~mm}$ pixel size, $110 \mathrm{kvp}, 3.6$ second and $4.71 \mathrm{~mA}$ was used. First and last reconstructions were performed using Image workNNT viewer version 2.21. All collected data were analyzed using SPSS.16 software. The mean, standard deviation and range were calculated for all land marks. For analysis of the qualitative data, Spearman correlation test was used. Data were classified by differences between BMI groups. Independent t-test and one way analysis of variance (ANOVA) were used for statistical analysis. $\mathrm{P}<0.05$ was considered statistically significant.

\section{Results}

Pearson correlation coefficient test was used for evaluating correlation between FSTT and BMI (Table 1). The test showed that there was a positive correlation between landmarks and BMI except for inferior malar, upper lip margin and supraorbital. LSD test was used for evaluating FSTT in BMI group (Table 2). There were significant differences between three BMI groups in supraorbital, occlusal line, zygion, glabella, supraglabella, suborbital and supraorbital point and the average of FSTT in patients with BMI up to 25 was more than patient with BMI of 20-25 and less than 20. The average of FSTT in patients with BMI of 20-25 was more than patient with $\mathrm{BMI}$ of less than 20 $(P<0.05)$. The inferior malar landmark was significantly different between the average of FSTT and three groups of BMI and the average in patient with BMI less than 20 was more than patent with $\mathrm{BMI}$ of $20-25$ and up to $25(\mathrm{P}<0.05)$.

\section{Discussion}

Imaging the head and neck region has been made easier than ever thanks to the emergence of the new generation of compact CT scanners (6) which are considered superior to the conventional multislice CT owing to their lower radiation dose, lower cost and higher special resolution (7).

Using cone beam computed tomography, Fourie et al. evaluated accuracy and reliability of facial soft tissue depth measurements. They suggested that face CBCT images could not only 
be utilized reliably for measuring the facial soft tissue thickness in region but also provide proper representation of the facial soft tissue (1).

To obtain correct facial reconstruction of human skull it is necessary to know the average facial soft tissue thickness of specific sites of the face. This requires establishing a database of soft tissue thickness related to ethnicity, weight and height. The present study determined the facial soft tissue thickness in the different landmark points on the CBCT images of patients $(8,9)$. In other studies, midline landmarks or mid-face landmarks were measured. In this study, we measured mid-face landmarks as well; mid-face region is the most important region for recognition $(2,4$, and 10$)$. Based on this study, in some landmarks significant differences were observed in underweight, normal and overweight subjects. In most of them, FSTT increased with increased BMI.

Dong et al. suggested that multiple factors including nutritional status should be taken into consideration. According to their study, soft tissue thickness measurements increased with increased BMI levels for both sexes at each landmark, and at most of the landmarks, statistically significant differences were observed between the different BMI subgroups (11). Codinha et al. emphasized on both the necessity of data presentation considering the sexual dimorphism, and introducing BMI as a pivotal variable for achieving more proper results of the likeness of the deceased individuals in both two-dimensional and three- dimensional facial approximations or superimpositions (12). Ward and Starbuck could determine the effect of body weight changes on face recognition accuracy by obese, normal and thin faces reconstruction in a skull and using data of soft tissue thickness (9). Sahni and colleagues studied facial soft tissue thickness in North-West Indian adults on MRI images. The patients with head trauma history or lesion and over or underweight patients were excluded from the study. There were significant relations between facial soft tissue thickness and height in two landmarks and significant differences between facial soft tissue thickness and weight in ten landmarks. They concluded that these data could be used in forensic science for facial reconstruction (5). In our study, inferior malar landmark thickness decreased when BMI increased.

\section{Conclusion}

In some landmarks, FSTT was associated with significant differences in underweight, normal and overweight patients. Often, FSTT increased when BMI increased. The results of this study could be used for facial reconstruction in Iranian population; however, slight differences between the studied population and other sub-group races most be considered for the accurate reconstruction. 

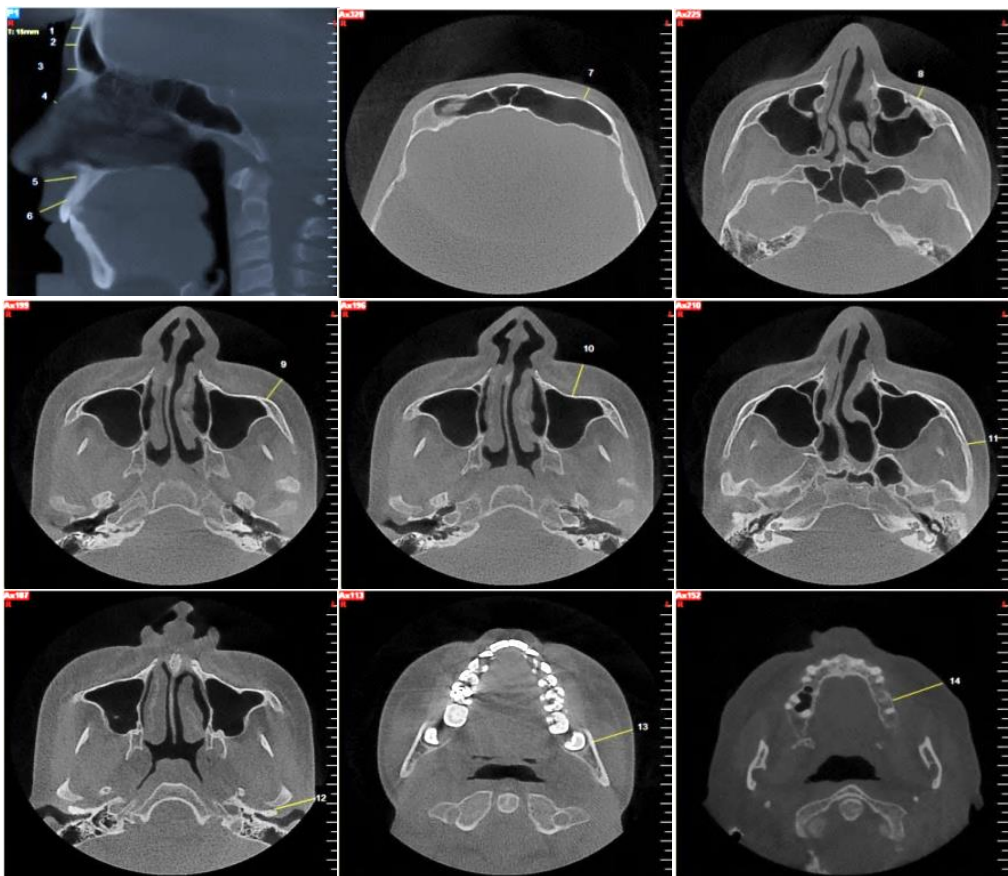

Figure 1: Anatomical landmarks in CBCT image

1, supraglabella; 2, glabella; 3, nasion; 4, rhinion; 5, midphiltrum; 6, upper lip margin; 7, supraorbital; 8 , suborbital; 9 , lateral orbit; 10 , inferior malar; 11, zygion; 12 , supraglenoid; 13 , occlusal line; 14 , supra M2

\begin{tabular}{ccc}
\hline landmarks & Sex & \\
\hline Supra glabella & $\mathrm{P}^{\text {-value }}{ }^{*}$ & correlation \\
Glabella & $\mathrm{P}<0.001$ & 0.448 \\
Nasion & $\mathrm{P}<0.001$ & 0.408 \\
Rhinion & 0.002 & 0.195 \\
Midphiltrom & 0.018 & 0.149 \\
Upper lip margin & 0.003 & 0.188 \\
Supra orbital & 0.097 & 0.104 \\
Sub orbital & $\mathrm{P}<0.001$ & 0.429 \\
Lateral orbit & $\mathrm{P}<0.001$ & 0.258 \\
Inferior malar & $\mathrm{P}<0.001$ & 0.229 \\
Zygion & 0.969 & -0.002 \\
Supraglenoid & $\mathrm{P}<0.001$ & 0.414 \\
Occlusal line & 0.923 & -0.006 \\
supra_M2 & $\mathrm{P}<0.001$ & 0.469 \\
& $\mathrm{P}<0.001$ & 0.361 \\
\hline
\end{tabular}

Table 1: Pearson correlation coefficient test 


\begin{tabular}{|c|c|c|c|}
\hline landmarks & BMI group & $p$-value & Mean deviation \\
\hline & $(20-25) \&(20>)$ & -0.72 & 0.005 \\
\hline \multirow[t]{3}{*}{ Supra glabella } & $(25<) \&(20>)$ & -1.62 & $P<0.001$ \\
\hline & $(25<) \&(20-25)$ & -0.89 & $P<0.001$ \\
\hline & $(20-25) \&(20>)$ & -0.58 & 0.036 \\
\hline \multirow[t]{3}{*}{ Glabella } & $(25<) \&(20>)$ & -1.42 & $P<0.001$ \\
\hline & $(25<) \&(20-25)$ & -0.83 & $P<0.001$ \\
\hline & $(20-25) \&(20>)$ & -1.06 & 0.082 \\
\hline \multirow[t]{3}{*}{ Midphiltrom } & $(25<) \&(20>)$ & -1.66 & 0.006 \\
\hline & $(25<) \&(20-25)$ & -0.59 & 0.062 \\
\hline & $(20-25) \&(20>)$ & -0.98 & 0.003 \\
\hline \multirow[t]{3}{*}{ Supra orbital } & $(25<) \&(20>)$ & -1.59 & $P<0.001$ \\
\hline & $(25<) \&(20-25)$ & -0.97 & $P<0.001$ \\
\hline & $(20-25) \&(20>)$ & 0.04 & 0.906 \\
\hline \multirow[t]{3}{*}{ Sub orbital } & $(25<) \&(20>)$ & -0.72 & 0.048 \\
\hline & $(25<) \&(20-25)$ & -0.76 & $P<0.001$ \\
\hline & $(20-25) \&(20>)$ & 0.35 & 0.612 \\
\hline \multirow[t]{3}{*}{ Lateral orbit } & $(25<) \&(20>)$ & -1.24 & 0.068 \\
\hline & $(25<) \&(20-25)$ & -1.59 & $P<0.001$ \\
\hline & $(20-25) \&(20>)$ & 6.59 & 0.003 \\
\hline \multirow{3}{*}{ Inferior malar } & $(25<) \&(20>)$ & 5.27 & 0.015 \\
\hline & $(25<) \&(20-25)$ & -1.32 & 0.248 \\
\hline & $(20-25) \&(20>)$ & -1.53 & 0.001 \\
\hline \multirow[t]{3}{*}{ Zygion } & $(25<) \&(20>)$ & -2.42 & $P<0.001$ \\
\hline & $(25<) \&(20-25)$ & -0.89 & $P<0.001$ \\
\hline & $(20-25) \&(20>)$ & -1.78 & 0.013 \\
\hline \multirow[t]{3}{*}{ Occusal line } & $(25<) \&(20>)$ & -4.10 & $P<0.001$ \\
\hline & $(25<) \&(20-25)$ & -2.31 & $P<0.001$ \\
\hline & $(20-25) \&(20>)$ & -1.45 & 0.123 \\
\hline \multirow{2}{*}{ Supra M2 } & $(25<) \&(20>)$ & -3.78 & $P<0.001$ \\
\hline & $(25<) \&(20-25)$ & -2.33 & $P<0.001$ \\
\hline
\end{tabular}

Table 2: LSD test used for evaluating FSTT in BMI groups

\section{Refrences}

1. Fourie Z, Damstra J, Gerris PO, Ren Y. (2010) Accuracy and reliability of facial soft tissue depth measurements using cone beam computer tomography. Forensic Sci. Int. 199, 9-14.

2. Panenkova P, Benus R, Masnicova S, Obertova Z, Grunt J. (2012) Facial soft tissue thicknesses of the mid-face for Slovak population. Forensic Sci. Int. 220,293-293.

3. Hwang H-S, Park M-K, Lee W-J, Cho J-H, Kim B-K, Wilkinson C.M. (2012) Facial Soft Tissue Thickness Database for Craniofacial Reconstruction in Korean Adults. J. Forensic Sci. November 57(6), 1442-1447.

4. Sipahioglu S, Ulubay H, Diren H.B. (2012) Midline facial soft tissue thickness database of Turkish population: MRI study. Forensic Sci. Int. 219,282-282.

5. Sahni D, Jit I, Singh P, Suri S, Sanjeev A, Kaur H. (2002) Preliminary study on facial soft tissue thickness by magnetic resonance imaging in Northwest Indians. Forensic Sci. Commun. 4(1).

6. P. Sukovic, Cone beam computed tomography in craniofacial imaging, Orthod. Draciofac. Res. 6 (2003) 179-182. 
7. V. Kumar, j. Ludlow, L.H.S. Cevidanes, A. Mol, In vivo comparison of conventional and cone beam CT synthesized cephalograms, Angle 78 (2005) 873-878.

8. Kim K.D, Ruprecht A, Wang G, Lee J.B, Dawson D.V, Vannier M.V. (2005) Accuracy of facial soft tissue thickness measurements in personal computer-based multiplanar reconstructed computed tomographic images, Forensic Sci. Int. 155,28-34.

9. Starbuck J.M, Ward R.E. (2007) theeffect of tissue depth variation on craniofacial reconstructions. Forensic Sci. Int. 172,130-136.

10. Rhine J.S, Moore C.E. (1984) Tables of Facial Tissue Thickness of American Caucasoids in Forensic Anthropology. Maxwell Museum Technical Series, vol. 1, University of New Mexico.

11. Dong Y, Huang L, Feng Z, Bai S, Wu G, Zhao Y. (2012) Influence of sex and body mass index on facial soft tissue thickness measurements of the northern Chinese adult population. Forensic Sci. Int. 222,396.e1=396.e7.

12. Codinha S. (2009) Facial soft tissue thicknesses for the Portuguese adult population. Forensic Sci. Int.184, 80-81. 